\title{
About the Existence of Integrable Solutions of a Functional-Integral Equation (l)
}

\author{
G. EMMANUELE
}

ABSTRACT. We improve (in some sense) a recent theorem due to Banas and Knap ([2]) about the existence of integrable solutions of a functional-integral cquation.

\section{INTRODUCTION}

Let $l=[0,1]$ be. We consider the following functional-integral equation

$$
x(t)=g(t)+f\left(t \cdot \int_{0}^{\dagger} k(t, s) x(\varphi(s)) d s\right) \quad t \in 1
$$

where $f: \mathrm{I} \times \mathrm{R} \rightarrow \mathrm{R}^{+}=[0,+\infty), k: \mathrm{I} \times \mathrm{I} \rightarrow \mathrm{R}^{+}, g: \mathrm{I} \rightarrow \mathrm{R} \varphi: \mathrm{I} \rightarrow \mathrm{I}$ are functions verifying special hypotheses (see section 2 ) and we look-for solutions $x \in L^{\prime}$ (I). As remarked in the paper [2] this equation has been considered by a number of authors because of its importance in problems in physics, engineering and economics; further, problems in the theory of partial differential equations lead, sometimes, to the study of the equation (1). Recently, Banas and Knap ([2]) gave a result of existence of integrable solutions to (1). They were forced by the techniques used to consider certain monotonicity assumptions on $g, f, k$ (see hypotheses i), ii) and iv) in [2]), that we are able to eliminate completely here. However, we must observe that Banas and Knap obtain a monotone solution, a fact that doesn't follow from our hypotheses. Prof Banas also observed that under our hypotheses we don't need to use the

(1) Work performed under the auspices of G.N.A.F.A. of C.N.R. and partially supported by' M.U.R.S.T. of Italy $(60 \%)$

1980 Matrematics Subject Classilication (1985 revision): $47 \mathrm{H} 15$. Editorial de la Universidad Complutense. Madrid. 1991. 
measure of weak noncompactness he considered in [2] because the operator we define following [2] actually has a relatively weakly compact range. So it is enough to apply, Tychonoff fixed point. Theorem ([5]). We take this opportunity to thank him very much for this remark that made our proof simpler.

\section{PRELIMINARIES AND MAIN RESULT}

As in the paper [2] we define the following four operators

$$
\begin{gathered}
(K x)(t)=\int_{1}^{1} k(t, s) x(s) d s \\
(F x)(t)=f(t, x(t)), \\
(H x)(t)=f\left(t, \int_{0}^{1} k(t, s) x(s) d s\right) \\
x=A x=g+H x(\varphi)=g+F K x(\varphi) .
\end{gathered}
$$

We consider the following hypotheses

(i) $g \in \mathrm{L}^{\prime}(1)$

(ii) $f: \mathrm{I} \times \mathrm{R} \rightarrow \mathrm{R}^{+}$satisfies Caratheodory hypotheses (i.e. $f$ is measurable with respect $10 t \in \mathrm{I}$, for all $\mathrm{x} \in \mathrm{R}$, and continuous in $x \in \mathrm{R}$, for a.a. $t \in \mathrm{J}$ ) and there are $a \in \mathrm{L}^{\prime}(\mathrm{I}), b \geq 0$ such that

$$
f(t, x) \leq a(t)+b|x| \quad t \in 1 ; \quad x \in \mathbf{R}
$$

(this last inequality is a necessary and sufficient condition for $F$, and so $H$, to take values in $L^{\prime}$ (I) when acting on elements of $L^{\prime}(I)$; see Theorem I in [2])

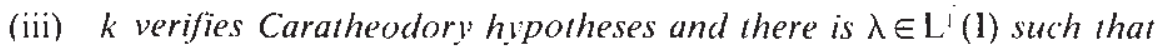

$$
k(t, x) \leq \lambda(t) \quad \text { ta.e. in } I, x \in \mathrm{R}
$$

(under (iii) the linear operat or $K$ maps $L^{\prime}(I)$ into $L^{\prime}(I)$ continuously; let us denote by $\|K\|$ its operator norm)

(iv) $\varphi: 1 \rightarrow I$ is absolutely continuous and there exists $B>0$ such that $\varphi^{\prime}(\mathrm{t}) \geq B$ for a.a. $t \in \mathrm{l}$.

(v) $\mathrm{b}\|K\| / B<1$ 
The technique used in [2] is the following: under the above assumptions $A$ is a weakly continuous operator from a suitable $B_{s}$ into itself; furthermore there exists $L \in[0,1]$ such that $\beta(A(Y)) \leq L \beta(Y),(\beta$ the measure of weak noncompactness introduced in [3]), for all nonempty subsets $Y$ of $B_{s}$ and hence results from [1] and [6] can be applied to get a fixed point of the operator $x \rightarrow g+F K x(\varphi)$. The difference between the result in [2] and our Theorem below resides in the technique we use to obtain the weak continuity of $A$ : indeed, Banas and Knap consider some monotonicity hypotheses on $g, f, k$ we are able to dispense with. Further, we do not make use of the measure of weak noncompactness introduced in [3] as remarked in the Introduction.

Theorem. Under the assumptions i)-v) above the equation [1] has at least a solution $x \in \mathrm{L}^{\prime}(\mathrm{I})$.

Proof. As in the paper [2] we can prove that $A: B_{s} \rightarrow B_{s}$, where $s=(\|g\|+\|a\|) /\left(t-b\left\|K^{\prime}\right\| B^{-1}\right)$. Furthermore, it is not difficult to see that the set $A\left(B_{\mathrm{s}}\right)$ is relatively weakly compact ([5]), since it is bounded and uniformly integrable. Hence Tychonoff fixed point Theorem ([5]) will conclude the proof once we have the weak continuity of $A$. So, we need only to show that $A$ is weakly continuous from $B_{s}$ into $B_{s}$, i.e. $A$ maps weakly convergent nets $\left(x_{x}\right) \subset B_{s}$ into weakly convergent nets $\left(A\left(x_{x}\right)\right)$. It is clearly enough to show that $H$ is weakly continuous. So let $\left(x_{\alpha}\right), x_{0} \subset B_{s}$ be with $x_{\alpha} \stackrel{M}{a} x_{0}$; if we prove that for any $\epsilon>0$, any $y^{*} \in \mathrm{L}^{*}(\mathrm{l}),\left\|y^{*}\right\| \leq 1$ and any subnet $\left(x_{\alpha_{\beta}}\right)$ of $\left(x_{\alpha}\right)$, there is another subnet $\left(x_{\alpha_{\beta_{\gamma}}}\right)$ for which $\mid<H\left(x_{\alpha_{\beta \gamma}}\right)$ $-H\left(x_{0}\right), y *>\mid<\epsilon$ we are done (proceeding by contradiction, of course).

To reach our target, we start by noting that the operator $x \rightarrow x(\varphi)$ from $\mathrm{L}^{\prime}(\mathrm{I})$ into itself is bounded and linear; hence it is weakly continuous and so $x_{\alpha}(\varphi)^{\prime \prime \prime} \rightarrow x_{0}(\varphi)$ in $\mathrm{L}^{\prime}(\mathrm{I})$. Since $B_{s}$ is bounded in $\mathrm{L}^{\mathrm{J}}(\mathrm{I})$, the set $\left\{x_{c k}(\varphi), x_{0}(\varphi)\right\}$ is even bounded in $\mathrm{L}^{\prime}(1)$, by a number $M$. Now, given $\epsilon>0$ choose $\delta>0$ such that meas (D) $<\delta$, implies $\int_{\underline{1}} 2[a(t)+b \lambda(t)] d t<\frac{\epsilon}{2}$. Furthermore, choose a closed subset $I_{1} \subset I$, meas $\left(I \backslash I_{1}\right)<\frac{\delta}{4}$, with $\lambda_{l_{1}}$ continuous (use Lusin Theorem, [4]) $Q=\max _{\mathrm{I}_{1}} \lambda$. Again consider a closed subset $\mathrm{I}_{2} \subset \mathrm{I}$, meas $\left(\mathrm{II}_{2}\right)<$ $\frac{\delta}{4}$, with $f_{\mathrm{I}_{2} \times[-Q M, Q M]}$ continuous (and so uniformly continuous) and a closed subset $I_{3} \subset 1$, meas $\left(I \backslash I_{3}\right)<\frac{\delta}{4}$, with $k_{l_{3} \times 1}$ continuous (and so uniformly continuous) (use Scorza-Dragoni Theorem, $[6]$ ). Put $I_{0}=\bigcap_{i=1}^{3} 1_{i} I_{0}$ is a closed subset of 1 . Now, observe that, for $t^{\prime}, t^{\prime \prime} \in \mathrm{I}_{0}$, if $\psi_{\alpha}(t)=\int_{0}^{+r^{i=1}} k(t, s) x_{\alpha}(\varphi(s)) d s$, $\psi_{0}(t)=\int_{0}^{1} k(t, s) x_{0}(\varphi(s)) d s$, one has 


$$
\left|\psi_{v g}\left(t^{\prime}\right)-\psi_{\alpha}\left(t^{\prime \prime}\right)\right| \leq \int_{0}^{1}\left|k\left(t^{\prime}, s\right)-k\left(t^{\prime \prime}, s\right)\right|\left|x_{\alpha}(\varphi(s))\right| d s
$$

(the same is true for $\psi_{0}$ ). Since $k_{\left.\right|_{1, x \mid}}$ is uniformly continuous and $\left(x_{k}\right) \subset B_{*}$, the set $\left\{\psi_{\alpha}, \psi_{0}\right\}$ is equicontinuous in $\mathrm{C}^{0}\left(\mathrm{I}_{0}\right)$. It is very easy to see that the same set is bounded by $Q M$ in the norm of $\mathrm{C}^{\prime \prime}\left(\mathrm{I}_{0}\right)$, hence the Ascoli-Arzelà Theorem can be applied to get a relatively compact subset of $C^{\prime \prime}\left(\mathrm{l}_{0}\right)$. The net $\left(\psi_{\psi_{\psi_{\beta}}}\right)$ admits a converging subnet $\left(\psi_{\alpha_{\alpha_{\gamma}}}\right)$. On the other' hand; for $\left.\bar{i} \in \mathrm{I}_{1}\right)$,

$$
\psi_{\alpha}(\bar{l})=\int_{0}^{l} k(\bar{t}, s) x_{t r}(\varphi(s)) d s \rightarrow \psi_{0}(\bar{l})=\int_{1}^{1} k(\bar{l}, s) x_{0}(\varphi(s)) d s
$$

since $x_{t z}(\varphi) \stackrel{n t}{\rightarrow} x_{0}(\varphi)$ in $\mathrm{L}^{\prime}(\mathrm{I})$ and $s \rightarrow k(\bar{f}, s)$ is in $\mathrm{L}^{\infty}(1)$. Hence $\psi_{x_{b_{z}}} \rightarrow \psi_{0}$ in the $\mathrm{C}^{0}$-norm on $\mathrm{I}_{0}$. Now, recall that $f_{\left.\left.\right|_{\mathrm{I}_{1} \times \mid-Q M} Q M\right]}$ is uniformly continuous and
so we have

$$
\lim _{\gamma} f\left(t, \psi_{u_{\beta_{\gamma}}}(t)\right)=f\left(t, \psi_{0}(t)\right) \text { uniformly on } \mathrm{I}_{0}
$$

Now, take $y^{*} \in \mathrm{L}^{\infty}(\mathrm{I})$, with $\left\|y^{*}\right\|_{\infty} \leq 1$, calculate this $y^{*}$ on $\left(f\left(\bullet, \psi_{t x_{\gamma_{v}}}(\bullet)\right)\right.$ $\left.-f\left(\cdot, \psi_{0}(\cdot)\right)\right)$

$$
\begin{aligned}
& \mid \int_{0}^{t} y^{*}(t)\left\{f\left(t, \psi_{\alpha_{\beta_{\gamma}}}(t)\right)-f\left(t, \psi_{0}(t)\right) d t \mid \leq\right. \\
& \leq \int_{l_{0}}\left|y^{*}(t)\right|\left|f\left(t, \psi_{\alpha_{\beta_{\gamma}}}(t)\right)-f\left(t, \psi_{0}(t)\right)\right| d t+ \\
& +\int_{\backslash_{1}}\left|y^{*}(t)\right| f\left(t, \psi_{\alpha_{\beta_{\gamma}}}(t)\right)-f\left(t, \psi_{0}(t)\right) \mid d t \leq \\
& \leq \int_{l_{0}}\left|f\left(t, \psi_{\alpha_{\beta_{\gamma}}}(t)\right)-f\left(t, \psi_{0}(t)\right)\right| d t+\int_{\Lambda \backslash_{0}} 2[a(t)+b \lambda(t)] d t .
\end{aligned}
$$

Now, recall that (2) is true and observe that

$$
\text { meas }\left(I \backslash \mathrm{I}_{0}\right) \leq \sum_{i=1}^{3} m\left(\mathbb{I} \backslash \mathrm{I}_{i}\right) \leq \frac{3}{4} \delta<\delta \text { so that } \int_{\backslash \backslash \mathrm{I}_{11}} 2[a(t)+b \lambda(t)] d t<\frac{\epsilon}{2} \text {. }
$$

Hence the last member of the chain of inequalities written above is smaller than $\epsilon$ for $\gamma$ sufficiently large. This is what we need to show that $H$ is weakly continuous on $B_{s}$. We are done. 


\section{References}

[1] J. Aprei1., E. De PAscat IE, Su alcuni parametri connessi con la misura di non compattezza di Hausdorff in spazi di funzioni misurabili, Boll. Un. Mat. It. (6) 3-B (1984) 497-515.

[2] J. ВАیАs; Z. KNAP, Integrable solutions of a functional-integral equation, Revista Matemática de la Univ. Complutense de Madrid, 2(1) (1989) 31 -38.

[3] F. S. DE BI.Asi, On a property of the unit sphere in a Banach space. Bull. Math. Soc. Sci. Math. R. S. Roumanie 21 (1977) 259-262.

[4] N. Dincuiliniu, Vector Measures. Pergamon Press 1967.

[5] N. Dusfok1, J. T. SchwARTy, Linear operators, part. I, Interscience 1958.

[6] G. EMmanuiz.e, Measure of weak nontompactness and fixed point theorems, Bull. Math. Soc. Sci. Math. R. S. Roumanie 25 (1981) 253-258.

[7] G. SCORzn-DrAgoni, Un teorema sulle funzioni continue rispetto ad una e misurabili rispetto ad unaltra variabile, Rend. Sem. Mat. Univ. Padova 17 (1948) 102-106.

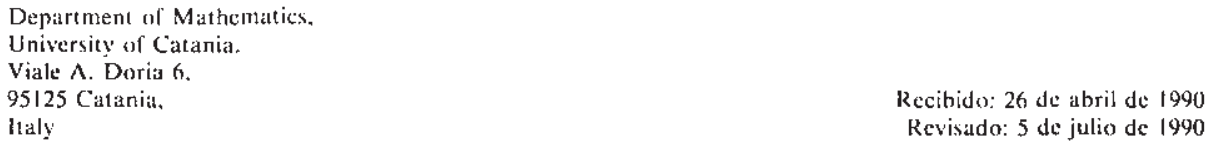

